\title{
An Embedded Internet of Energy Communication Platform for the Future Smart Microgrids Management
}

\author{
S. Marzal, R. González-Medina, R. Salas-Puente, G. Garcerá, Senior Member IEEE, E. Figueres, \\ Senior Member, IEEE.
}

\begin{abstract}
Microgrids are moving toward electric power systems in a sort of an internet of energy (IoE) where a large number of generators can be connected anywhere. In this regard, to realize the envisioned IoE, information and communication technologies (ICT) are crucial for developing innovative applications and services as well as achieving high levels of efficiency in microgrids. However, due to the variety of ICT, there is not a de facto standard solution to implement IoE platforms. Moreover, standards for the current Internet of Things (IoT) platforms are not optimal for developing IoE platforms, which present more demanding challenges. In such context, this paper presents an embedded IoE platform for management of smart microgrids. The performance of this platform has been tested in an experimental microgrid. Results show that the proposed platform fulfills the microgrids requirements and it is able to manage the energy flows, the safety issues, etc. in microgrids.
\end{abstract}

Index Terms-Microgrids, Internet of Energy, ICT, embedded technology

\section{INTRODUCTION}

$\mathrm{T}$ he current and expected future needs of energy demand along with the challenge of gradual electric power supply decarbonization, demand the search for alternative, cleaner and renewable energy sources. From this point of view, Microgrids (MGs) have been raised as a potential solution for the integration of these renewable and distributed energy resources in the energy system. A microgrid can be defined as a low-voltage distributed network composed by Distributed Generators (DG), Energy Storage Systems (ESSs), local loads and power electronic interfaces, which can operate in either grid connected and island modes [1].

The integration of Distributed Energy Resources (DERs) into MGs is traditionally carried out by means of centralized control systems, in which a central controller communicates with all the microgrid resources, receiving information about its state and taking the control decisions. However, the recent increase in the number of DERs that are deployed into microgrids provokes that centralized communication topologies are not the optimal solution for monitoring and controlling the microgrid resources. In effect, these topologies

Manuscript received Month xx, 2xxx; revised Month xx, xxxx; accepted Month $\mathrm{x}, \mathrm{xxxx}$. This work is supported by the European Regional Development Fund (ERDF) under Grant ENE2015- 64087- C2-2 R. This work is supported by the Spanish Ministry of Economy and Competitiveness (MINECO) under BES2013-064539. could lead to inefficiencies in the communication network, even the fault or the shutdown of the global system if the central control point fails [2]. To overcome this limitation, decentralized communication topologies have been proposed for microgrids. In these topologies, an improvement of the microgrid reliability is achieved by removing the central controller as a single point of failure. Following the decentralized approach, the local nodes have their own Microgrid Control System (MCS) and, therefore, have the ability to take decisions. This is the key issue that features Smart Microgrids (SMGs), i.e., the added intelligence including bidirectional communications, sensing and management capabilities. In addition to in time detecting and reacting to events, these features allow the ability to maintain high levels of efficiency, reliability, and Quality of Service (QoS). Is in this scenario where the concept of Internet of Energy (IoE) can be interesting (Figure 1). Compared with the traditional microgrid, the IoE allows connected devices (DERs) become globally accessible and real-time controllable, anytime, anywhere [2], [3], [4].

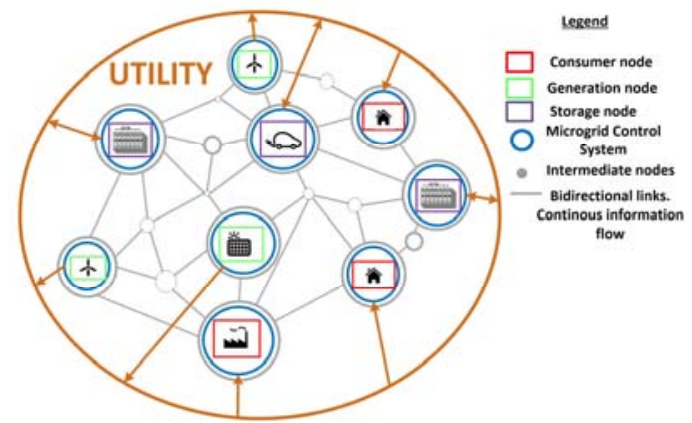

Fig.1.Overview of the future IoE Smart Microgrid Scenario

The Internet of Things (IoT) can integrate objects to the Internet. To achieve that, the IoT infrastructure is composed by embedded smart devices, with limited computational resources, which are able to provide certain real time services, such as monitoring, communications, controlling, among others [5], [6]. This idea can be extended to IoE which deals to

S.Marzal, R. González-Medina, R.Salas-Puente, G. Garcerá and E. Figueres are with Grupo de Sistemas Electrónicos Industriales del Departamento de Ingeniería Electrónica, Universitat Politècnica de València, Camino de Vera s/n, 46022 Valencia, Spain; raugonme@upv.es; rosapue1@posgrado.upv.es ; ;ggarcera@eln.upv.es, efiguere@eln.upv.es.

* Correspondence: silmarro@upv.es; Tel.: +34-96-3877007 (ext. 76021) 
interconnect distributed energy resources by means of the Internet [7]. The structure of the Internet of Things is mainly based on Peer-to Peer (P2P) networks composed by autonomous nodes [8]. It is worth to point out that P2P communications networks have been mainly developed for applications such file and processor cycle sharing, whose network performance requirements are less critical (in the order of seconds) than microgrid functions (in the order of milliseconds). Moreover, normally IoT networks have an excessive dependence on the existing Internet data center service [9], [10]. This fact could produce delays in dispatching services, provoking undesirable effects in the microgrid. Therefore, the current IoT software and related communication platforms are not properly adapted for microgrids environments due to QoS requirements for microgrids are particularly demanding [11]. Moreover, although the IoE concept can be applied to SMGs, there is not a standard solution for its implementation [12]. In fact, the current microgrids development does not use the IoE approach [13].

To overcome this limitations, this paper proposes a novel IoE platform and distributed embedded systems that implement real-time interfaces between the microgrid and the cloud. The platform is oriented to monitoring and control purposes and is able to fulfill the demanding performance requirements of microgrids.

The main scientific contributions of the paper are summarized as follows:

1) The design and development of a flexible, distributed and embedded IoE communication platform based on P2P communications which can effectively collect, process and analyze the microgrid data, from anywhere and anytime, for monitoring and control purposes.

2) An innovative IoE-P2P middleware which is able to work in both synchronous and asynchronous mode, to guarantee the choice of the proper and optimal type of communication for each task or communication messages that are interchanged into the microgrid. To our knowledge, currently there is not any communication IoE platform that involves synchronous and asynchronous $\mathrm{P} 2 \mathrm{P}$ distributed communications.

3) An IoE protocol and a routing algorithm specifically designed for monitoring and control of SMGs, which is able to fulfill the demanding SMGs requirements.

4) To implement a real IoE platform the DERs have been embedded into devices with limited computational resources. The efficient use of the computational resources in order to guarantee the required SMGs performance is experimentally proved.

5) To evaluate the proposed IoE infrastructure in terms of QoS, it has been deployed in an experimental microgrid becoming, at least to our knowledge, the first experimental IoE communication platform for managing SMGs.

The rest of this paper is organized as follows; Section II describes the challenges for developing IoE communication platforms. In Section III the proposed communication platform is described. In Section IV some experimental results are presented and discussed. Finally, Section V provides the conclusions of this study.

\section{Challenges of the InTERnet OF ENERGY}

The Internet of Energy is an emerging paradigm that applies IoT technologies to energy control systems [14]. Today, IoE introduces significant new challenges for developing advanced communication infrastructure for SMGs [13], [15]. To better understanding of the challenges that face the IoE, the main differences between Internet of Energy and traditional microgrids are described as follows [8], [16], [17]:

1. The architecture: In contrast with traditional microgrids, the IoE architecture is highly integrated with an information network. In today's microgrids, the information system and the physical system are independent. In contrast to this, the information system is vital for the $\mathrm{IoE}$ due to it allows the interaction with the physical system. By combining both physical and information networks, the IoE aims to optimize the energy utilization on the basis of safe and stable operation.

2. The access of Information: A microgrid is formed of locally distributed energy resources in order to improve reliability. In contrast, Internet of Energy aims at comprehensive energy optimization, which consists on the autonomous access to multiple DERs. In the Internet of Energy there is not a centralized hardware or administrative nodes in the network, thus the nodes have the same hierarchy and roles.

3. The information and energy exchange: The IoE demands equal, free and real-time energy and information sharing, which can be achieved by using address and routing capabilities, avoiding faults in case of nodes failures. This is not limited to the primary path and backup paths as traditional microgrids do.

4. The embedded capabilities: Unlike traditional microgrid, the distributed energy resources deployed on the microgrid become controllable anytime and anywhere. Each node has embedded and "plug and play" capabilities, so if damage or fault is caused at any node, this event will not affect to the operation of the whole system.

Related to the architecture, the IoE, as any Internet of Thing (IoT) platform, has a structure based on four layers: Things, Network, Service and Cloud. With this architecture, networking, data transfer and applications are highly integrated.

The access of information is another challenge in IoE. Traditionally, communication architectures for microgrids use a client-server based communication topology for information accesses. In this approach the data center plays a central role to process the petitions and manage the information, which means that the DERs have little autonomy. Differently, in the IoE, each distributed energy resource should act as both clients (master) and server simultaneously [18]. The kind of networks that allow this functionality are called P2P networks. In these networks, the connectivity between the nodes is carried out by creating virtual links, overlays, which are built on top of the physical network which allow more flexibility than physical networks, extensibility, self-healing and dynamic reconfiguration. Therefore, create virtual links that allow nodes self-management in order to make local adaptive decisions on the basis of the information they receive from the agents to which they are linked is a big challenge to achieve the IoE.

One of the major challenges to realize the envisioned $\mathrm{IoE}$ is the information and energy exchanges in real-time. One of the 
features of the IoE is its massive scale. In particular, a large number of devices can be connected to the microgrid producing continuous flows of data that must be processed in real-time [19]. Since microgrids are complex distributed systems, the IoE infrastructure needs a direct, efficient and in time communication to meet the demanding microgrid network performance requirements (in the order of few milliseconds). According to the IEC 61850 and IEEE 1646/1647 standards the typical response time for different SMG functions should follow the values in Table I [20], [21], [22].

$$
\text { TABLE I }
$$

NETWORK REQUIREMENTS FOR MGS

\begin{tabular}{cc}
\hline Function & Response Time \\
\hline Monitoring Information & $15 \mathrm{~ms}-200 \mathrm{~ms}$ \\
Control Information & $16 \mathrm{~ms}-100 \mathrm{~ms}$ \\
Messages requiring immediate actions & $3 \mathrm{~ms}-100 \mathrm{~ms}$ \\
Distribution Management & $100 \mathrm{~ms}-2 \mathrm{~s}$ \\
Demand Response & $500 \mathrm{~ms}-$ several minutes \\
\hline
\end{tabular}

Besides, energy systems are becoming increasingly complex, so it is vital to build flexible architectures with a high capacity to exchange information in a networking environment that is in continuous evolution. In such an environment some quality properties, like efficiency, mobility support, adaptability, reliability, and timeliness, are required [23]. The fulfilment of these requirements is achieved by middleware solutions, located in the service layer, mainly based on the Request/Response (RR) or the Publish/Subscribe (PubSub) paradigms. In the first case, RR is a communication scheme based on one-to-one communication interaction. Senders deliver request messages to specific receivers that process and reply the request. This message exchange follows a synchronous method invocation, i.e., to guarantee the message delivery, the connection remains open until the responses arrive. However, the difficulties for decoupling time and space make that the RR paradigm is not well suited for mobility support, since the requester can be indefinitely blocked while waiting for a response. In the second case, PubSub is a nonblocking asynchronous communication scheme where the distribution of events is one-to-many. The providers of information (publishers) disseminate events to the system and the information consumers (subscribers) are subscribed to the events in which they are interested. Nevertheless, this method has difficulty guarantying a reliable delivery due to the decoupling between subscribers and publishers. It is worth pointing out that conventional middleware platforms have been developed by implementing only one of the two communication paradigms [23]. However, middleware solutions for microgrids need simultaneously using RR and PubSub-based communication mechanisms [12], [23], [24]. The reasons for that are, on the one hand, that in a microgrid the control information among DERs require a communication system based on query-response. In this regard, a timely response is needed for carrying out immediate actions, due to if the communication delay exceeds the required time, the information does not fulfill its purpose and, in the worst case, damage might occur. On the other hand, for microgrid monitoring tasks, PubSub paradigm is needed. For example, when a load is switched from off to on, an event could be notified to other DERs to evaluate the global status of the microgrid. Another challenge related to middleware is that $\mathrm{IoE}$ devices can be shared by several IoE services or applications. This fact may lead to resource conflicts and transient overloads that should be solved by the middleware. Therefore, the interaction management between devices that is carried out by the middleware should involve new forms of routing based on logical criteria, such as: the type of DERs device, the subscriptions or communities of interest, the geographical proximity, etc. Summarizing, middlewares for microgrids need to support both RR and Pub-Sub communications paradigms due to each communication paradigm provides orthogonal functionalities and different quality properties. Table II defines the contribution of each communication paradigm to the proposed IoE platform.

TABLE II

PROPERTIES PROMOTED BY EACH COMMUNICATION PARADIGM

\begin{tabular}{|l|c|c|}
\hline \multicolumn{1}{|c|}{ Property } & RR & PubSub \\
\hline Efficiency & Partial & Partial \\
\hline Adaptability & - & $\bullet$ \\
\hline Security & $\bullet$ & Partial \\
\hline Reliable delivery & $\bullet$ & - \\
\hline Timeliness & $\bullet$ & - \\
\hline Mobility & - & $\bullet$ \\
\hline
\end{tabular}

Legend: $\bullet$ Indicates that the characteristic is available in the category specified in the column heading. - Indicates that it does not

By definition, in the IoE, any network-connected device is an embedded system. Hence, an IoE device has limited computing and storage resources. In conventional IoT, devices often operate in a single-thread context without parallel processing support [12]. This means that tasks are sequentially processed. However, in microgrids this is not optimal due to a critical task might not be in time because the resources are busy by performing other tasks. So, a crucial challenge for The IoEplatform is to develop embedded software in each node, which should be able to manage multiple thread and parallel processing with minimum waste of computational resources. Moreover, due to the virtual layer of the $\mathrm{P} 2 \mathrm{P}$ architectures, Plug and Play (P\&P) capabilities are allowed and when the peers join the network, peers communicate with each other to establish dynamic self-organizing structures on top of the underlying physical networks.

To transform traditional systems into IoE, all these challenges must to be faced. The working line for developing IoT-based energy systems are outlined below.

With regard to the challenges that have been described above, several studies can be found in the literature. In [14], [25] the requirements of IoE are provided. However, the infrastructures have not been developed and neither simulation analysis nor experimental results about the performance of the IoE platforms have been carried out. In [13], [26], [27], [28], [29], [30], [31],[32], [33] several IoT software infrastructures that enable energy management are presented. In these studies, only simulation results have been carried out so the main practical issues related to the deployment of a real platform are not treated. Moreover, they neither involve P2P distributed networks nor the integration of the different communications paradigms. A major issue related to the application of the IoE concept to microgrids is the development of hardware allowing the practical deployment of this kind of networks. In this sense, special attention should be paid, among other aspects, to the use of embedded devices, the influence of the electromagnetic 
noise on the communication quality and the practical verification of performance requirements.

In summary, a practical IoE platform should provide:

1) High availability for large-scale systems that support P2P communications,

2) Flexible communications platforms which allow RR and PubSub communications paradigms,

3) Provide quality performance attributes and efficient routing

3) High level programming to embed the software into the DERs.

\section{The Proposed IoE COMMUNICATION PlatForm}

Building a distributed IoE platform for energy management is a challenging task. The tasks that must been executed by the platform are: (i) the integration of the $M G$ devices in the communication network, and (ii) the interconnection of the devices among them and with the IoE cloud for monitoring and control purposes. To carry out these tasks the proposed IoE communication platform is composed by 4 layers, as shown in Figure 2. A description of each layer is provided below.

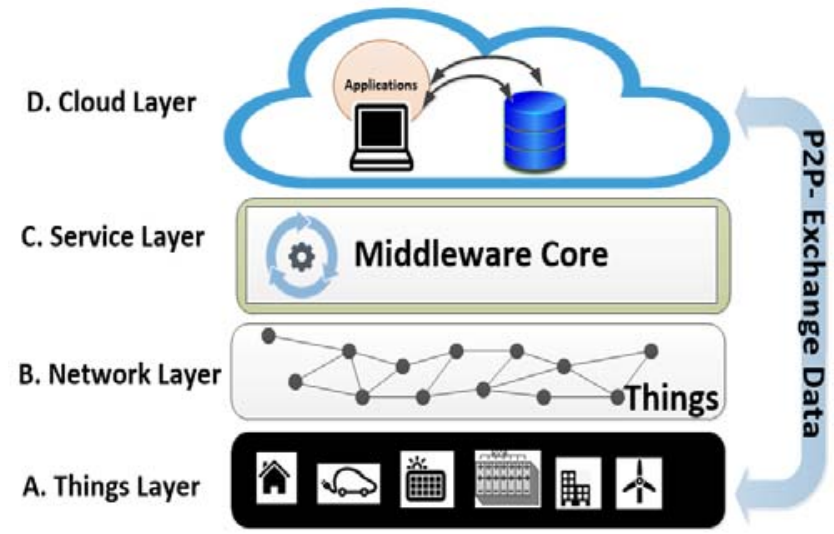

Fig.2. Overview of the proposed IoE platform architecture

A sample schema of this platform is depicted in Figure 3. It is composed by the following components:

(A) The things layer which integrates the available microgrid hardware to control/sense the status of the things

(B) The network layer which defines the protocols and networks used for connect the things

(C) The service layer which creates and manages services according to the things needs. This layer relies on the middleware technology which provides messaging and routing layer which support run-time switching between RR and PubSub communications paradigms to integrate microgrid services and functionalities in IoE.

(D) The cloud layer which includes the IoE application. This layer is at the top of the architecture and is responsible to data store and data analysis. Application layer comprise of the custom Microgrid applications that is making use of the things data.

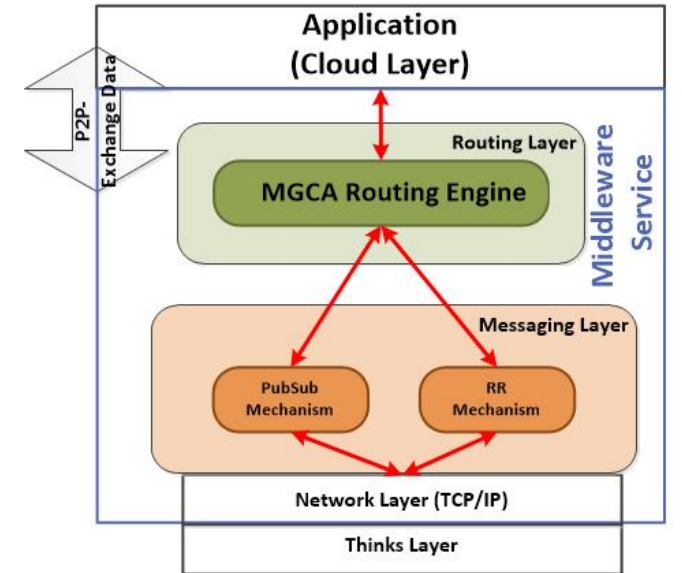

Fig.3. Simplified schema of the proposed IoE platform architecture

\section{A. Things Layer}

Following the IoT/IoE terminology, each DER of the microgrid is tagged like a thing. Therefore, DERs must incorporate an Intelligent and Communicative Electronic Device (IED). There are two communication interfaces in this layer. The first interface communicates each DER with the power network and it is carried out by means of UART serial communications. Modbus is the chosen serial protocol to implement this interface, due to it is an open protocol that requires low computer resources; in addition, it defines a message structure that DERs can recognize and use. The second interface links each DER with the distributed communication network. In this case, a P2P communication network over Ethernet is the chosen technology due to the advantages described in Section II.

\section{B. Network Layer}

The network layer is the infrastructure that allows things to manage the communication in the distributed network and transmit messages between things and the service layer. In this layer, TCP/IP are the chosen protocols due to these protocols are the standard for the Internet. They enable end-to-end communications independently of the underlying physical media, things and network layer. This characteristic provides interoperability among third-party non-compliant end devices and compliant communications networks. This characteristic provides interoperability among third-party non-compliant end devices and compliant communications networks, improving flexibility. Network interoperability is indispensable to achieve an overall optimal system operation and connectivity, independently from the chosen physical medium, the type of devices and manufacturers.

\section{Service Layer}

In this layer, the middleware is considered a key technology for developing efficient IoE applications [3], since this layer provides the interconnection between things and the cloud layer. An essential issue is the accomplishment of the network requirements for MGs and the appropriate and reliable message delivery. It is therefore vital to develop a middleware able to provide QoS in terms of latency, delivery rates and bandwidth, among other factors. In order to fulfill these requirements, the 
proposed middleware is formed by two layers: Messaging layer and Routing layer.

\section{1) Messaging Layer}

As it has been outlined in Section II, the integration of RR and PubSub communication mechanisms in microgrids is needed. Each communication paradigm provides orthogonal functionalities and different quality properties. With Request/Response communication pattern, quality properties as reliability, timeliness and security are achieved. In addition, efficiency, mobility support and adaptability are quality properties achieved by PubSub architecture. Both communication systems have been developed by the authors in [34] and [35] respectively. Therefore, this subsection is only focused on the integration of RR and PubSub. It is worth pointing out that both systems are integrated in the middleware but they are not simultaneously working. The proposed mechanism to automatically select RR or PubSub as communication system is based on the type of exchanged message. Table III shows the type of messages and their associated communication system that have been defined for the proposed IoE platform.

TABLE III

INTERCHANGED MESSAGES ON THE PROPOSED IOE PLATFORM

\begin{tabular}{|c|c|c|}
\hline $\begin{array}{l}\text { Type of } \\
\text { message }\end{array}$ & Description & $\begin{array}{c}\text { Comm. } \\
\text { Type }\end{array}$ \\
\hline $\begin{array}{c}\text { Peers } \\
\text { Commands }\end{array}$ & Lookup, Join, Leave, Discovery & PubSub \\
\hline $\begin{array}{l}\text { Monitoring } \\
\text { Information }\end{array}$ & $\begin{array}{c}\text { Power Consumed, State of Charge } \\
\text { (SoC), Micro Generation, Voltage, } \\
\text { Frequency, etc. }\end{array}$ & $\begin{array}{c}\text { RR/ } \\
\text { PubSub }\end{array}$ \\
\hline $\begin{array}{l}\text { Control } \\
\text { Information }\end{array}$ & $\begin{array}{l}\text { Flows of energy: Grid to MG, MG to } \\
\text { Grid, Energy Dispatch Limit, Load } \\
\text { Limits, MG On/Off, etc. }\end{array}$ & $\mathrm{RR}$ \\
\hline $\begin{array}{l}\text { Critical } \\
\text { Messages }\end{array}$ & $\begin{array}{l}\text { Messages that require immediate } \\
\text { actions }\end{array}$ & RR \\
\hline $\begin{array}{c}\text { Distribution } \\
\text { Management }\end{array}$ & Peers coordination actions & RR/PubSub \\
\hline $\begin{array}{l}\text { Demand } \\
\text { Response }\end{array}$ & $\begin{array}{l}\text { Non priority data (energy market, } \\
\text { peers data, etc.) }\end{array}$ & PubSub \\
\hline Status & $\begin{array}{c}\text { Failure, Notifications } \\
\text { Messages } \\
\end{array}$ & PubSub \\
\hline Stabilize & Node Reports & PubSub \\
\hline
\end{tabular}

In this regard, a specific microgrid communication protocol has been developed for transmitting data among things. In the proposed protocol, a set of attributes associated with each delivered message are defined. These attributes are: (i) type of message, which identifies the communication mechanism (RR or PubSub), (ii) iD Device, which identifies the type of DER (Critical Load (CL), Non Critical Load (NCL), Photovoltaic Generator (PVG), Energy Storage System (ESS), etc.), (iii) subscription code, only in case of PubSub messages, which identifies the events in which subscribers are interested and (iv) data parameters and values, which are transmitted into the P2P network. Figure 4 shows an overview of the implemented microgrid communication packet structure. The developed message packet frame (MSG Packet Frame) follows a fairly uniform overall structure which contains a header, data, and footer. The header and the footer fields contain several bytes of control information, which is used to communicate important facts about the data that the message contains and how it is to be interpreted and used. The data that should be transmitted (payload of message) is composed by the attributed listed above

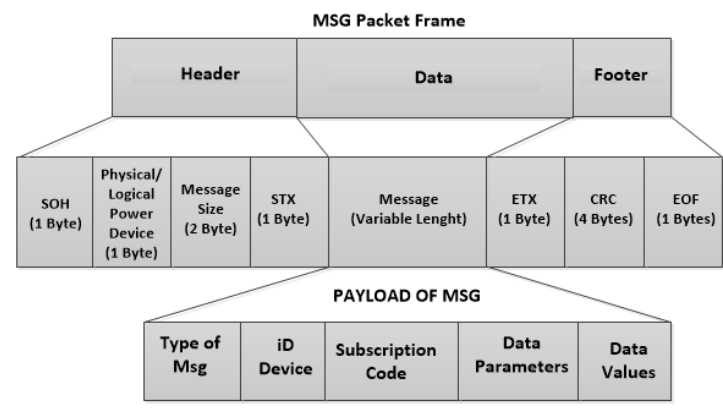

Fig.4. Overview of Packet Frame Structure

When a packet is sent, the proper method (RR or PubSub) is selected. Then, the packet is transmitted to the target things, accordingly to the routing algorithm that is explained below. The target things process the packet based on the communication type.

\section{2) Routing Layer}

P2P networks generates high rates of network traffic which can lead to high delays, poor bandwidth utilization, packet loss, wasted computer resources, among other inefficiencies. This is due to, traditionally, flooding techniques are used in $\mathrm{P} 2 \mathrm{P}$ networks for routing. In flooding routing schemes, when a node receives a query, it forwards it to all the nodes of its routing table and the nodes recursively retransmit the query. The flooding stops after TTL $=4$ hops. The lookup message will visit each node at least one time to obtain data retrieval.

To avoid unnecessary retransmissions and reduce the network traffic to meet the microgrid network performance requirements, a microgrid clustering algorithm (MGCA) has been developed to carry out the messages routing. Clusters are logical groups of things in form of communities to achieve common objectives [36]. Thus, in this work, the routing is based on transmitting the information only to the selected clusters instead of broadcasting the information to all the things.

The proposed MGCA is a content-based algorithm. In this approach, the logical clustering is formed depending on the active communication type (RR or PubSub). For RR routing, the clusters are formed following the DER functionality (i.e. its iD Device). In the case of PubSub routing, the things are grouped in clusters according to the subscription code. The chosen protocol to create the clusters has been a modified Chord Distributed Hash Table (DHT). The Chord DHT protocol is a well-known protocol for P2P networks [37] in which peers find other peers, and have access to their respective data, through a ring topology. Each peer in the ring upholds a finger table, which is a distributed hash table that stores the peer identifiers that are used by the lookup algorithm for routing. The finger table of the conventional Chord DHT has been modified in order to establish the communication only with the cluster of interest. To achieve this, the finger table needs to discriminate among the categories of clusters. Therefore, it embeds both the DER functionality and the subscriptions code. When a thing joins the peer network, it sends this information to the things neighbors. Thus, each thing has a modified routing table whose entries follow the form $\left(T o M, I D_{D E R}, \mathrm{SC}_{\mathrm{i}}, \mathrm{L}_{\mathrm{i}}\right)$, where ToM identifies the type of message (RR or PubSub), $I D_{D E R_{i}}$ identifies the DER functionality, $\mathrm{SC}_{\mathrm{i}}$ stands for a subscription code, and $\mathrm{L}_{\mathrm{i}}$ is a link that contains the IP address. 
With this modification of the finger tables, the clustering process can be done by means of the MGCA algorithm. This algorithm searches the things that are inside of the cluster of interest accordingly to the DER functionality (with RR) or the subscription codes (with PubSub). When the matched things are identified, a message with the things match list will be sent to the thing that has started the request.

Based on the described above, the Microgrid Cluster Algorithm is presented in Algorithm 1. The algorithm uses an overlay Chord-graph ranged from ranged from 0 to $2^{m}-1$, where $m$ is the number of bits in the identifiers.

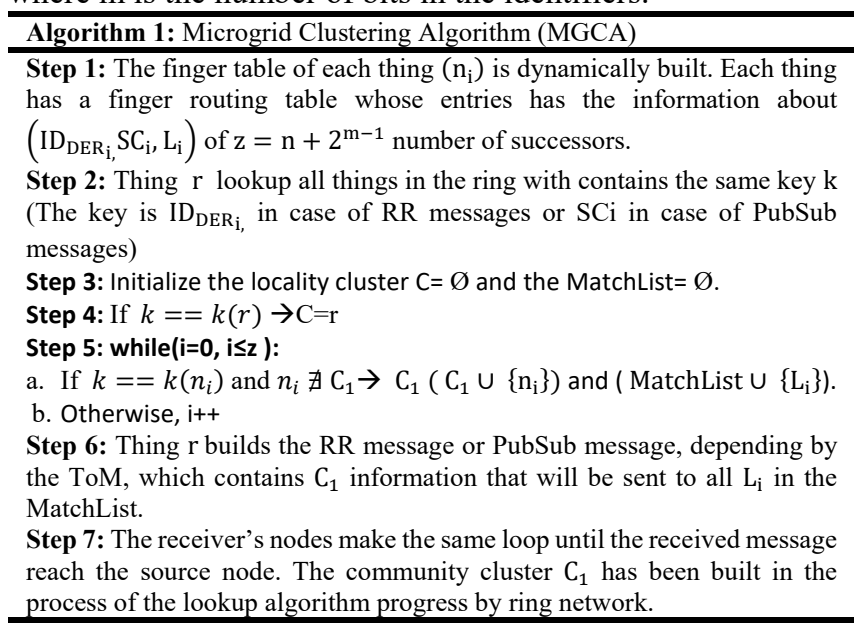

It should be note that Cluster $C_{1}$ has all locality nodes of searched key type $\left(I D_{D E R}\right.$, in case of RR messages or $\mathrm{SC}_{\mathrm{i}}$ in case of PubSub messages). The MatchList is composed by all IPs of nodes which the message will be sent. In this way, this thing can establish the communication only with the things that are included in that list, avoiding sending unnecessary messages and reducing the latencies and the used bandwidth.

\section{Cloud Layer}

The cloud layer stores the historical data that has been obtained from the DERs for global supervision purposes. Storing historical data is one of the required features for IoE applications and services [38]. The cloud layer of the IoE platform contains virtualized servers. Moreover, an application interface with stored historical data for each DER has been implemented. The historical database is able to store and manage a huge amount of data, which is provided to the cloud infrastructure by the application interface.

\section{APPLICATION TO THE MICROGRIDS MANAGEMENT: A CASE EXAMPLE}

To illustrate the above concepts, it has been considered a residential microgrid composed by loads, photovoltaic generation and storage devices (ESS). The microgrid under consideration is managed following the photovoltaic generation profile and the so-called Time of Use (ToU) tariffs. The possibility of connecting both critical (CL) and non-critical loads (NCL) has been taking into account in this example. From the management point of view, the main difference between CL and NCL is the higher priority of CL if the available energy is low. NCL will be considered as constant load that will be connected or fully disconnected if there is not enough energy to supply it. Instead, CL is not disconnected but the supplied power is limited according to the available energy and the applicable tariff. This is a general approach that is compatible with the particular case of having the need of fully supplying CL independently from the tariff (i.e. if there is not enough PV generation, the needed energy would be demanded to the grid assuming the corresponding costs).

TOU tariffs are a new concept designed to incentivize costumers to use more energy at off-peak times. Normally these tariffs are classified in Peak, Shoulder and Off-Peak periods, being the peak tariff the most expensive and the off-peak the cheapest. Only for illustrating purposes, Figure 5 shows the considered tariffs along the day.

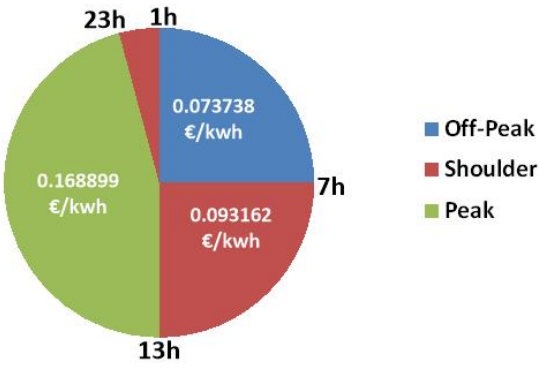

Fig.5. Applied TOU periods

A ToU-algorithm has been developed to dynamically manage the flows of energy into the microgrid according the load profile, the State of Charge (SoC) of EES, the available PV generation and the applicable tariff. Figure 6 shows the flowchart of the algorithm with the set of rules that has been taken into account. The main guidelines are described below: 1) The hired power (HP) is the maximum power that can be consumed from the main grid. The maximum power that can be supplied to the loads (PLim) is the sum of the photovoltaic generation (PV), the hired power (HP) and the power that can be extracted from the batteries (PBat). Therefore, the power consumed by the loads can be greater than HP only if photovoltaic production is available or there is enough energy stored in the batteries.

2) It has been considered a tariff with time discrimination (peak and off-peak stretches). Therefore two values have been established for HP, corresponding to each one of the stretches. The energy supplied by the main grid in the peak stretch is much more expensive, so HP is significantly reduced in this stretch.

3) Two types of loads have been considered: a constant power non-critical load (NCL) and critical loads (CL), whose consumption varies according to a typical consumption profile. NCL is activated only if there is enough available power and the battery charge (SOC) is greater than $40 \%$. NCL is deactivated as long as the battery charge is below $25 \%$. Finally, $\mathrm{CL}$ is always connected but Plim limits the supplied power.

4) The battery is only charged if there is surplus photovoltaic production. If the battery is fully charged, the surplus is injected into the grid.

Note that these set of rules are just an example of power management in the microgrid. These rules are the context to apply the proposed communication architecture, which is the main objective of this paper. In this way, the proposed IoE 
middleware is used for efficiently routing the interchanged messages. PubSub is used to notify the applicable ToU tariff to all DERs (or things) in the network and RR transmits the microgrid commands according to the restrictions that were outlined in Table I. The historical data is stored in the cloud layer to supervise the global status of the microgrid.

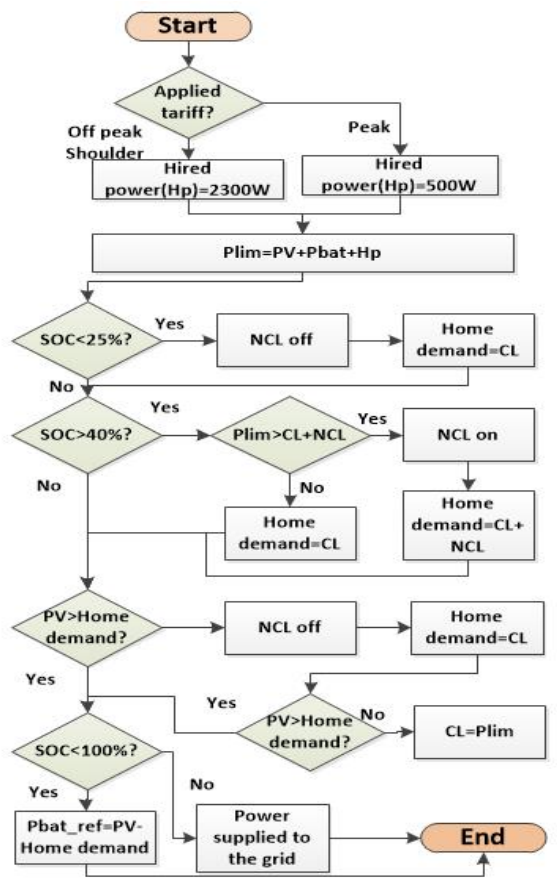

Fig.6. Flowchart of the TOU-Algorithm

\section{EXPERIMENTAL RESULTS}

To evaluate the performance of the proposed IoE platform, it has been applied to an experimental hybrid microgrids (HY$\mathrm{MG}$ ) in which there are two interconnected AC and DC buses [39]. As Figure 7 shows, the microgrid has six connection nodes, each one of them having a connected DER following table IV, (i.e. there is only a thing connected to each one of the nodes, so the terms nodes and things are equally used in the following). In this table, it has been described the kind of DER and the physical laboratory equipment that has been used to emulate it. Note that the performance of NCL and ILC has not been emulated. A $750 \mathrm{~W}$ resistor and $10 \mathrm{kVA}$ three phase inverter have been used to implement NCL and ILC, respectively.

A $400 \mathrm{~V}$ DC bus and a three-phase $230 \mathrm{~V}$ AC bus compose the power network. As it can be seen in Figure 8, each thing interacts with the power network and with the virtual distributed communication network through embedded single board computers (SBCs). The chosen SBC has been the Beagle Bone Black (BBB), which is a low-cost, small size and powerful SBC that is considered the best option for developing real-time IoT applications [26]. This board features an ARMCortex-A8 running at $1 \mathrm{GHz}$. Regarding the memory capacities, it integrates 512MB RAM and $2 \mathrm{GHz}$ onboard flash eMMC, which hosts a Debian 9 operating system. Moreover, it also includes GPIO ports, USB port, 10/100 Ethernet port and a HDMI connection port, among others functionalities. For these reasons, $\mathrm{BBB}$ has been used in this work as communication gateway to Ethernet-P2P network, as well as running platform to host the proposed IoE middleware.

Figure 7 shows a picture of the experimental setup, following the scheme that Figure 6 shows.

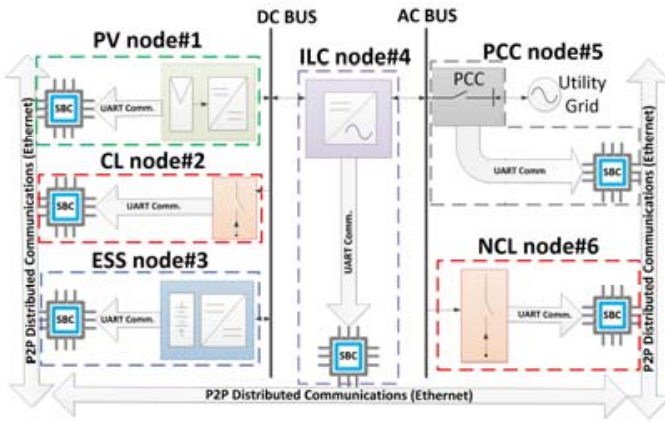

Fig.7. Conceptual hybrid microgrid diagram with six nodes and their communication interfaces.

It is worth noting that each node has an additional electronic board (EB) that works together with the corresponding SBC. The SBC provides connection to the virtual network via Ethernet, while the EB is only used for controlling the lab equipment to emulate the desired behavior of the connected DER, following Table IV. Obviously, there is not necessary an EB controller for the nodes in which the DER is not emulated (in this case, CL and ILC)

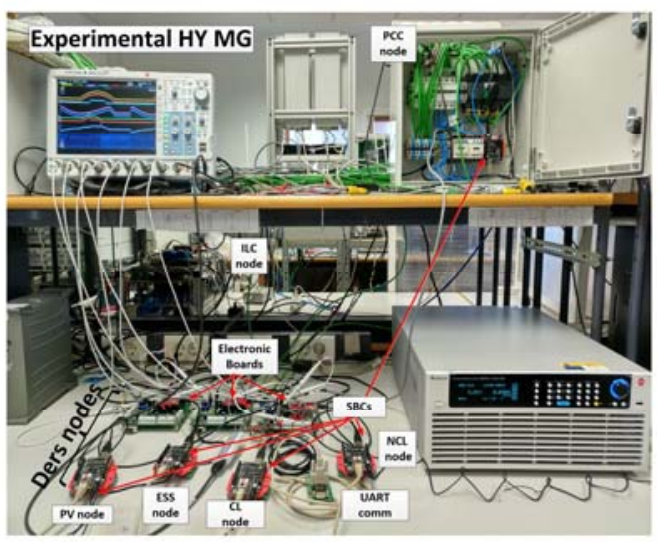

Fig.8. Picture of the experimental setup

In order to evaluate the proposed IoE communication platform, three tests have been carried out: A) IoE platform Response Time evaluation, B) IoE platform performance evaluation, C) Routing performance Evaluation, and D) IoE platform computational resources usage (on the embedded system) evaluation. 
TABLE IV

CHARACTERISTICS OF THE LAB EQUIPMENT

\begin{tabular}{|c|c|}
\hline Nodes & Lab Equipment \\
\hline$\# 1$ & $\begin{array}{l}\text { PV Generator, emulated by means of a DC programmable } \\
\text { power supply (AMREL SPS 800-12 DO13) }\end{array}$ \\
\hline$\# 2$ & $\begin{array}{c}\text { CL node, emulated by means a high power programmable } \\
\text { DC electronic load (Chroma } 63205 \mathrm{~A}-1200-200)\end{array}$ \\
\hline \#3 & $\begin{array}{c}\text { EES node, emulated by means a programmable } \\
\text { bidirectional DC power supply (REGATRON } \\
\text { TC.GSS.20.600.400.S.HMI) }\end{array}$ \\
\hline$\# 4$ & ILC node, a three phase inverter(10kVA) \\
\hline$\# 5$ & $\begin{array}{l}\text { PCC node, emulated by means an AC three phase power } \\
\text { source (Pacific, 360AMX). }\end{array}$ \\
\hline$\# 6$ & NCL node, a resistive load $(750 \mathrm{~W})$ \\
\hline
\end{tabular}

\section{A. IoE platform time-response evaluation}

To evaluate the response time of the communication system, it has been analyzed the transmission of an activation event for NCL. The experiment has been carried out by using both RR and PubSub paradigms. However, the response time of RR communications has a large impact on the overall message exchange, so the experiment has been focused on RR. With PubSub communications, the message exchange contains mostly unidirectional notification messages.

\section{A.1 Time Response of Request-Response Communications}

Figure 9 shows the communications exchange between two nodes during the NCL activation event using RR communication.

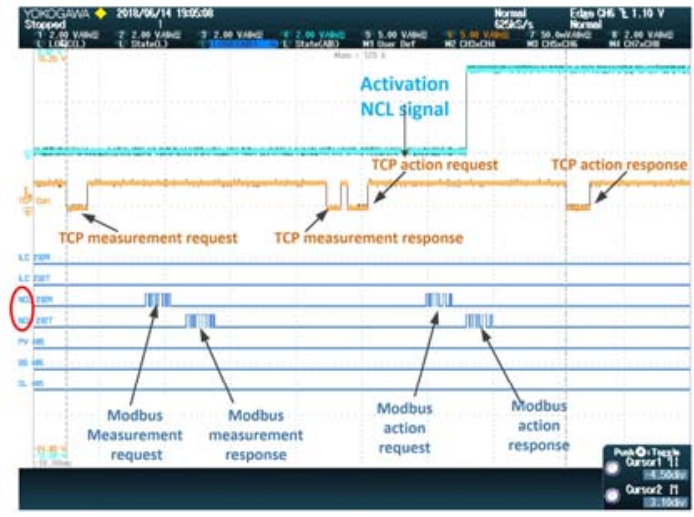

Fig.9. Communication exchanges to produce the NCL event activation

It is worth pointing out that, to carry out the different time measurements, the GPIO pins on Single Board Computers (SBC) and Electronic Boards (EBs) have been activated. Particularly, TLS pin (Transport Layer Security) for SBCs and the two pairs DTR/DSR (Data Terminal Ready/ Data Set Ready) RTS/CTS (Request to Send/Clear to Send) for EBs. Figure 10 shows the diagram with the signals activation related with the different communication exchanges.

Note that DTR/DSR-RTS/CTS pins have been used for data flow control to the devices (Modbus protocol activation). However TTL pin are mostly used for data flow control between the host and device (TCP protocol activation).

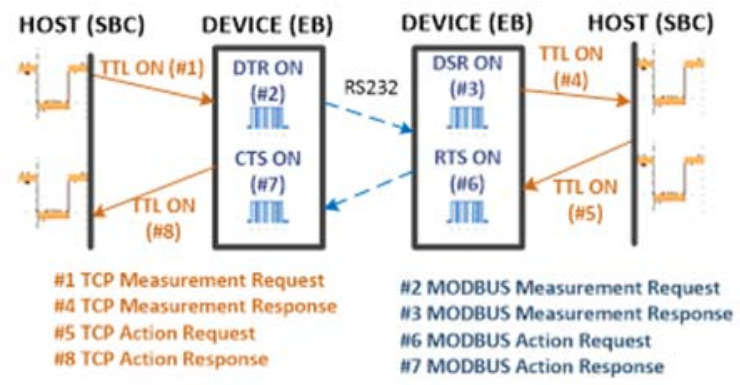

Fig.10. Diagram with the different GPIO signals activation for time request measurements

The communications exchanged during the NCL activation are the following: the source node of the P2P network request the state of the NCL node (TCP measurement request); when the NCL node in the network is reached (see the red circle in Figure 9), it reads its state through Modbus communication (Modbus measurement request) and responds (Modbus measurement response) by following TCP/IP protocol. When the source node is reached (TCP measurement response) it sends an $\mathrm{ON}$ action request (TCP action request); when this message arrives to the NCL (Modbus action request) it sends the ON signal and the NCL is activated (See NCL signal activation in blue). Finally, NCL sends an acknowledgement message (Modbus action response) through TCP/IP, which reaches the source node (TCP action response).

In this example the responses time of the different MG functions has been determined (See Figure 11)

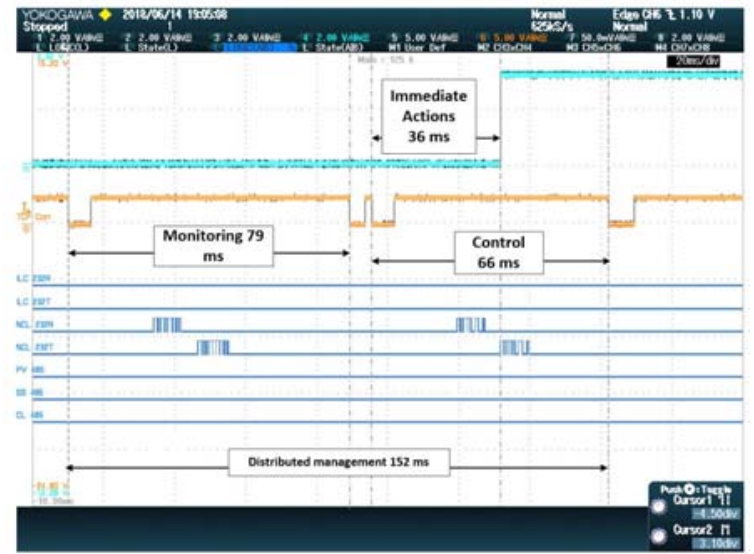

Fig.11. Response times of the exchanged messages by using RR communication

Table $\mathrm{V}$ defines the functions associated with the measured times. Table VI resumes the measured time-responses of each microgrid function with the proposed IoE platform, by comparing them to those required by microgrids. As it can be seen, the response time of the proposed IoE platform meets the network microgrid requirements. 
TABLE V

DEFINITION OF CONSIDERED RESPONSES TIMES

\begin{tabular}{|c|c|}
\hline Function & Definition \\
\hline Monitoring & $\begin{array}{c}\text { Time difference between the time instant when an } \\
\text { information request is send by the source node until } \\
\text { the response reaches the source node }\end{array}$ \\
\hline Control & $\begin{array}{c}\text { Time difference between the time instants when a } \\
\text { control request is send by the source until the control } \\
\text { ACK message is reached by node source }\end{array}$ \\
\hline $\begin{array}{c}\text { Immediate } \\
\text { actions }\end{array}$ & $\begin{array}{c}\text { Time difference between the time instants when an } \\
\text { action request is sent by the source node until the } \\
\text { action is executed. }\end{array}$ \\
\hline $\begin{array}{c}\text { Distributed } \\
\text { Management }\end{array}$ & $\begin{array}{c}\text { Time difference between the time instants when } \\
\text { information and an action request is sent by the } \\
\text { source node until the responses reach the source node. }\end{array}$ \\
\hline
\end{tabular}

TABLE VI

RESPONSE TIMES OBTAINED BY THE PROPOSED IOE PLATFORM

\begin{tabular}{ccc}
\hline Function & $\begin{array}{c}\text { IoE Platform Time } \\
\text { Response }\end{array}$ & $\begin{array}{c}\text { MG Demanding } \\
\text { Time Response }\end{array}$ \\
\hline $\begin{array}{c}\text { Monitoring } \\
\text { Information }\end{array}$ & $79 \mathrm{~ms}$ & $15 \mathrm{~ms}-200 \mathrm{~ms}$ \\
$\begin{array}{c}\text { Control Information } \\
\text { Messages requiring } \\
\text { immediate actions } \\
\text { Distribution }\end{array}$ & $66 \mathrm{~ms}$ & $16 \mathrm{~ms}-100 \mathrm{~ms}$ \\
Management & $36 \mathrm{~ms}$ & $3 \mathrm{~ms}-100 \mathrm{~ms}$ \\
\hline
\end{tabular}

Since in RR protocol, the requesting node keeps waiting for the response, a critical scenario (overload) has been considered to evaluate the platform's response time. For that, three hosts in broadcast have been requesting at once over the NCL node periodically every second during 10 minutes. The Response time between a minimum delay (best case), a medium delay (regular case) and a maximum delay (worst case) has been measured. Figure 12 shows the platform's response time under overload considering the delay time in the best, regular and worst case.

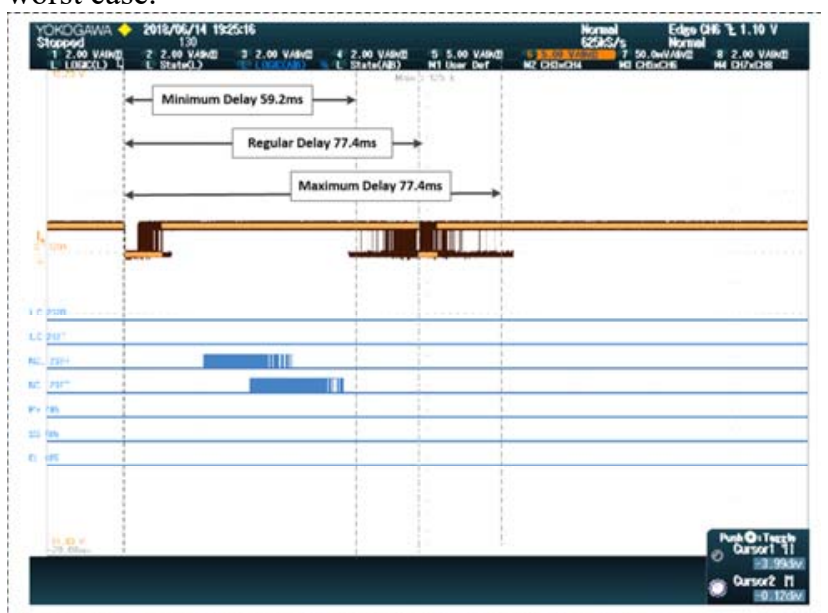

Fig.12. Response times under overload by using RR communication

As it can be seen, even in the worst case the response time of the proposed IoE platform meets the network microgrid requirements.

However, if a node failure occurs and the requester node does not receive the response of the destination node after three message retransmission, the node assumes that these nodes have left the network, and then update the list of its neighbors. It is worth to point out that, in normal conditions, the nodes communicate that they are leaving the network. Thus, in the case of node failure, it will not previously warn that leaves the network and the system will have proof that a failure has occurred. The subsequent management of failures is beyond the scope of this paper.

\section{A.2 Time Response of Publisher-Subscribe Communications}

The delays associated to the Pub-Sub communications have been evaluated by means of the Demand Response function. The Demand Response is a function that allows customers, voluntarily, to participate in load management. In table I, the required Demand Response Time (DRT) is in the range from $500 \mathrm{~ms}$ to several minutes. Since it is a specification relatively relaxed (several minutes without specifying a concrete superior limit), it has been implemented by means of a PubSub communication pattern. Figure 13 shows an example of the Demand Response function implemented through PubSub communication. In the example, the nodes CL (pink) and NCL (blue) are subscribed to a publication related to the loads activation/deactivation. The measurement of the DRT starts when the TCP/IP connection is stablished ( $\mathrm{t} 0$ ). As it can be seen in the figure, DRT $=1575 \mathrm{~ms}$ for CL disconnection and DRT $=1800 \mathrm{~ms}$ for activation of NCL. The measured times are clearly into the specified range.

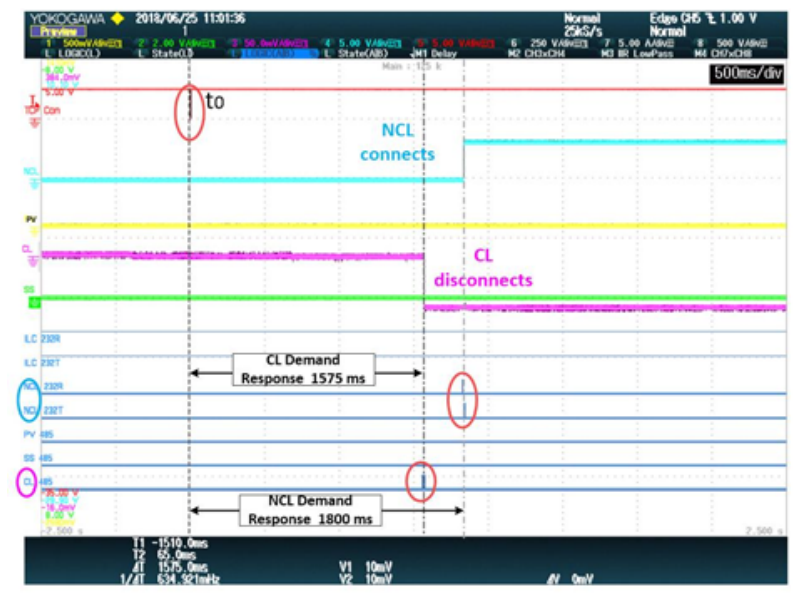

Fig.13. Demand response time by using PubSub communication

\section{B. IoE platform performance evaluation}

The goal of the proposed IoE platform is to support vertical applications that process the information from the things layer to the cloud layer. To evaluate the performance of the proposed IoE platform, the application example that was described in Section IV has been implemented on the experimental microgrid. The ToU-Algorithm that was introduced in that section (see Figure 6) has been deployed in each DER of the microgrid, running on top of the proposed IoE middleware. Figure 14 shows the behavior of each DER according to the programmed ToU-Algorithm and the set of communications that is taking place among the nodes of the microgrid. 


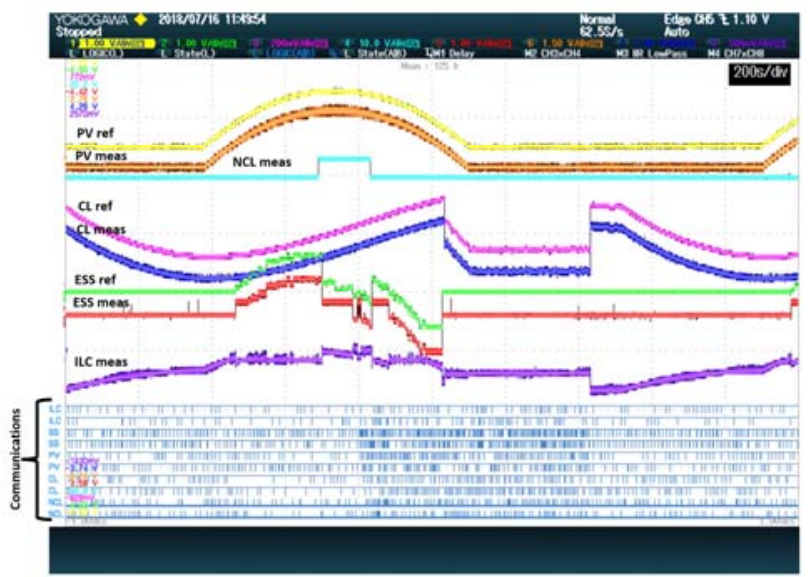

Fig.14. Set of IoE communications (down), control sígnals and flows of Energy (up) in the application example under consideration

The experiment emulates the behavior of the microgrid under study along a day (from $00 \mathrm{~h}$ to $24 \mathrm{~h}$ ). In the figure, it can be seen the reference signals for each node (i.e., the control signals to emulate the performance of the desired DER, which are labeled with the suffix "ref") as well as the power that is actually delivered or absorbed by the corresponding DER: PV generator (orange), NCL (light blue), CL (dark blue), ESS (red) and ILC (violet). It is worth noting that power is positive in loads when it is absorbed, but in the case of the PV generator and the ESS, it is assumed as positive when delivered. In the case of the ILC, the power is positive when the energy is flowing from the $\mathrm{DC}$ bus to the $\mathrm{AC}$ bus. It should be also noted that the reference signals are sent through the proposed IoE platform. As Figure 14 shows, the power that is being supplied/absorbed by each DER agrees perfectly with the corresponding reference signal. Therefore, the proposed IoE platform is efficiently working and all the commands are sent in a timely and reliable manner (see the communication packets at the bottom of the figure), verifying that the proposed IoE platform is adequate for the microgrids management.

\section{Evaluation of Routing Performance}

The main benefits of the proposed MGCA protocol are a reduction in both the network traffic (See Figure 15.a) and the routing time (See Figure 15.b). The performance of the proposed routing protocol has been compared with the one of flooding routing technique.

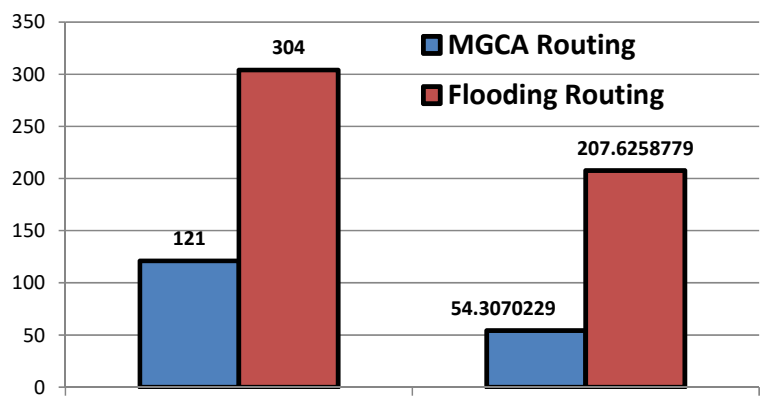

(a) Network Usage (KBps)

(b) Time Delay ( $\mu \mathrm{sec})$

Fig.15. Comparison of network usage in KBps (15.a) and time delay in microseconds (15.b) between MGCA and Flooding routing
The traffic in the network has been measured by averaging the network usage in kilobytes per second (KBps). In the same way, the average time delay is the average time to route the query to the matched nodes in microseconds. Both measurements have been evaluated for 6 nodes during 15 minutes.

\section{Performance Improvement (in \%)}

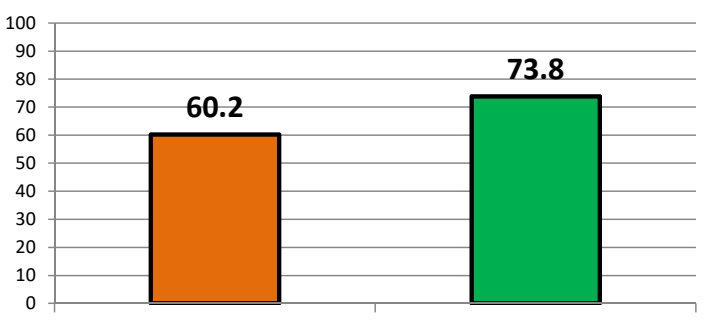

(a) Network Usage

(b) Time Delay

Fig.16. Improvement (in \%) of MGCA routing protocol over Flooding protocol

As Figure 16.a shows, the MGCA routing protocol improves near $60 \%$ the network usage. In other words, the proposed routing protocol consumes near three times less traffic that flooding techniques. The reason is that the routing tables of MGCA protocol have information about neighbors and clustering can be formed. In this way, each query is delivered only to the matched nodes in the cluster and not to the all nodes in the routing table as flooding routing does. Consequently, a network traffic reduction is achieved. In addition, MGCA protocol has self-organization capability due to the overlay Chord infrastructure. With this feature, each node knows the disposition of their neighbors, which produces an effective method for queries propagation. Figure 16.b shows a reduction around $73.8 \%$ in the routing time with MGCA with regard to the flooding protocol.

\section{Evaluation of computational resources usage}

Embedded systems work under several resources constraints as size, weight and consumed energy, among other limitations. As a consequence, they have limited memory and CPU capacities. Therefore, an efficient resource management is an essential aspect to integrate SBCs in real-time applications. In order to demonstrate that the proposed IoE middleware accomplish with the constraints of the chosen embedded hardware, it has been measured the usage of memory, as well as the percentage of $\mathrm{CPU}$, for processing of the proposed algorithms. To evaluate these parameters, they have been both measured with (IoE mode) and without (Idle mode) running the proposed IoE middleware. Obviously, the differences between the values that have been measured in both operation modes are the additional resources that the proposed IoE algorithm needs. As Figure 17 shows, the additional average CPU usage when the IoE algorithm is running is $4.35 \%$. Similarly, the additional average active memory usage is $10.6 \%$. It is worth noting that the needed computational resources are really far of the limits of the chosen SBC, so that other functionalities as storage of historical events, security protocols, etc. could be implemented. The implementation of such functionalities is out of the scope of this paper. 


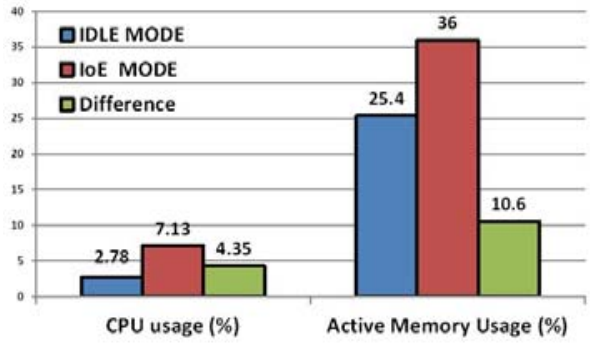

Fig. 17. Comparison of CPU and memory average use (in \% with regard to the full capacity of devices) between Idle and IoE modes

\section{CONCLUSIONS}

In this paper a new IoE communication platform for the management of microgrids has been presented. The proposed platform is based on a flexible IoT layered infrastructure that has highly scalability and integrates distributed energy resources (things layer). The platform is composed by four layers: things, IoE middleware, MG services and cloud. The platform has been physically implemented on an experimental hybrid-microgrid by means of Beagle Bone Black devices.

The achieved experimental results have shown that: 1) the IoE platform provides timely responses to events within precise timing constraints in order to guarantee a desired level of performance, 2) The proposed IoE platform operates efficiently for power management of residential applications and 3) The IoE platform is able to work properly under the restrictions of physical embedded systems.

Therefore, it can be concluded that the proposed solution is an interesting approach to the envisioned IoE in the context of microgrids. In addition, it may be easily extended to the Smart Grid concept.

\section{REFERENCES}

[1] González, R. M., Wattjes, F. D., Gibescu, M., Vermeiden, W., Slootweg, J. G., and Kling, W. L. "Applied internet of things architecture to unlock the value of smart microgrids", IEEE Internet of Things Journal.2016

[2] Barbierato, L., Estebsari, A., Pons, E., Pau, M., Salassa, F., Ghirardi, M., and Patti, E. "A Distributed IoT Infrastructure to Test and Deploy RealTime Demand Response in Smart Grids", IEEE Internet of Things Journal.2018

[3] G. Blair, D. Schmidt, and C. Taconet, "Middleware for Internet distribution in the context of cloud computing and the Internet of Things". Ann. Telecommun. vol.71, pp.87-92, 2016.

[4] F.G. Brundu, E. Patti, A. Osello, M. Del Giudic, et.al., " IoT Software Infrastructure for Energy Management and Simulation in Smart Cities", IEEE Trans. Ind. Infor., 13(2), 832-840. 2017.

[5] Rana, M. M., and Xiang, W. "IoT Communications Network for Wireless Power Transfer System State Estimation and Stabilization”, IEEE Internet of Things Journal, 5(5), 4142-4150.2018.

[6] Rana, M. M., Xiang, W., Wang, E., Li, X., and Choi, B. J. “ Internet of Things Infrastructure for Wireless Power Transfer Systems", IEEE Access, 6, 19295-19303.2018.

[7] Rana, M. M., Xiang, W., and Wang, E. 2IoT-Based State Estimation for Microgrids, IEEE Internet of Things Journal, 5(2), 1345-1346.2018.

[8] Su, W., and Huang, A. (Eds.). "The Energy Internet: An Open Energy Platform to Transform Legacy Power Systems into Open Innovation and Global Economic Engines". Woodhead Publishing.

[9] Li, J., Bao, Z., and Li, Z.,"Modeling Demand Response Capability by Internet Data Centers Processing Batch Computing Jobs". IEEE Trans. Smart Grid, 6(2), 737-747. 2015.

[10] Minoli, D., Sohraby, K., and Occhiogrosso, B. "IoT considerations, requirements, and architectures for smart buildings-Energy optimization and next-generation building management systems", IEEE Internet of Things Journal, 4(1), 269-283.2017.

[11] Jin, J., Gubbi, J., Marusic, S., and Palaniswami, M. "An information framework for creating a smart city through internet of things". IEEE Internet of Things journal, 1(2), 112-121.2014.

[12] V. Tanyingyong, R. Olsson, J.W. Cho, et.al., "IoT-grid: IoT communication for smart DC grids". In Global Communications Conference (GLOBECOM), 2016 IEEE (pp. 1-7).

[13] A. Q. Huang, M. L. Crow, G. T. Heydt, et.al., "The Future Renewable Electric Energy Delivery and Management System: The Energy Internet," Procs. of the IEEE, 99(1), 133-148, 2011.

[14] N. Bui, A.P. Castellani, P. Casari, and M. Zorzi, "The internet of energy: a web-enabled smart grid system”. IEEE Network, 26(4). 2012.

[15] M.M.Rana,"Architecture of the Internet of Energy Network: An Application to Smart Grid Communications", IEEE Access, 5, 4704-4710. 2016.

[16] Abe, R., Taoka, H., \& McQuilkin, D. (2011). Digital grid: Communicative electrical grids of the future. IEEE Transactions on Smart Grid, 2(2), 399410.

[17] Sun, Q. (2018). Energy Internet and We-Energy. Springer.

[18] Guerrero, J., Chapman, A. C., and Verbič, G. "Decentralized P2P Energy Trading under Network Constraints in a Low-Voltage Network", IEEE Trans. on Smart Grid. 2018

[19] Li, S., Yang, J., Song, W. Z., and Chen, A. "A Real-Time Electricity Scheduling for Residential Home Energy Management”, IEEE Internet of Things Journal.2018.

[20] International Electrotechnical Commission. IEC 61850-7-420 Communication Networks and System in Power Utility Automation IEEE: Piscataway, NJ, USA, 2009.

[21] IEEE Standard Communication Delivery Time Performance Requirements for Electric Power Substation Automation; IEEE Standards Association: Piscataway, NJ, USA, 2005; pp. 1-24.

[22] IEEE 1646 standard communication delivery time performance requirements for electric power substation automation; 2004.

[23] C. Rodríguez-Domínguez, K. Benghazi, M. Noguera, J.L. Garrido, M.L. Rodríguez and T. Ruiz-López, "A communication model to integrate the request-response and the publish-subscribe paradigms into ubiquitous systems". Sensors, 12(6), 7648-7668. 2012.

[24] C. Rodríguez-Domínguez, K. Benghazi, M. Noguera, J. L. Garrido, M.L. Rodríguez, and T. Ruiz-López, “A communication model to integrate the request-response and the publish-subscribe paradigms into ubiquitous systems", Sensors, 12(6), 7648-7668. 2012.

[25] Sun, Q., Zhang, Y., He, H., Ma, D., and Zhang, H. "A novel energy function-based stability evaluation and nonlinear control approach for energy internet". IEEE Trans. on Smart Grid, 8(3), 1195-1210. 2017

[26] J. Byun, I. Hong, and S. Park, "Intelligent cloud home energy management system using household appliance priority based scheduling based on prediction of renewable energy capability". IEEE Trans. on Consumer Electronics, 58(4), 2012.

[27] F. Lezama, J. Palominos, A. Rodríguez-González, A, Farinelli, and de E.M.Cote, "Agent-based microgrid scheduling: An ICT perspective". Mobile Networks and Applications, 1-17, 2017.

[28] F. G. Brundu, E. Patti, A. Osello, et.al., "IoT Software Infrastructure for Energy Management and Simulation in Smart Cities", IEEE Trans. Industrial Informatics, 13(2), 832-840. 2017.

[29] A. Maffei, S. Srinivasan, P. Castillejo, et.al., "A Semantic-MiddlewareSupported Receding Horizon Optimal Power Flow in Energy Grids", IEEE Trans. on Industrial Informatics, 14(1), 35-46. 2018.

[30] V. Tanyingyong, R. Olsson, J. W. Cho, et.al., "IoT-grid: IoT communication for smart DC grids", In Global Communications Conference (GLOBECOM), 2016 IEEE (pp. 1-7).

[31]Luo, X., Zhang, S., and Litvinov, E. "Practical Design and Implementation of Cloud Computing for Power System Planning Studies”, IEEE Trans. on Smart Grid. 2018.

[32] Bedi, G., Venayagamoorthy, G. K., Singh, R., Brooks, R. R., and Wang, K. C. "Review of Internet of Things (IoT) in Electric Power and Energy Systems". IEEE Internet of Things Journal, 5(2), 847-870. 2018.

[33] Zanella, A., Bui, N., Castellani, A., Vangelista, L., and Zorzi, M., "Internet of things for smart cities", IEEE Internet of Things journal, 1(1), 22-32.2014.

[34] S. Marzal, R. González-Medina, R. Salas-Puente, E. Figueres, and G. Garcerá, "A novel locality algorithm and peer-to-peer communication infrastructure for optimizing network performance in smart microgrids", Energies, 10(9), 1275. 2017. 
[35] S. Marzal, R. Salas-Puente, R. González-Medina, G. Garcerá, and E. Figueres. "Efficient Event Notification Middleware for Smart Microgrids over P2P Networks", IEEE Trans. on Smart Grid.2018

[36] Cenedese, A., Luvisotto, M., and Michieletto, G., "Distributed clustering strategies in industrial wireless sensor networks". IEEE Trans. on Ind. Infor, 13(1), 228-237.2017

[37] I. Stoica, R. Morris, D. Karger, M.F. Kaashoek, M.F.; H. Balakrishnan, "Chord: A scalable peer-to-peer lookup service for internet applications", Comput. Commun. Rev. 2001, 31, 149-160.

[38] C. Esposito, A. Castiglione, F. Frattini, M. Cinque, Y. Yang and K. R. Choo, "On Data Sovereignty in Cloud-based Computation Offloading for Smart Cities Applications," , IEEE Internet of Things Journal.2018

[39] Shahparasti, M., Mohamadian, M., Baboli, P. T., and Yazdianp, A. "Toward power quality management in hybrid ac-dc microgrid using ltc-l utility interactive inverter: Load voltage-grid current tradeoff', IEEE Trans. on Smart Grid, 8(2), 857-867. 2017. 